\title{
sciendo
}

\section{DO THE DIFFERENCES IN EGG CONTAMINATION, PENETRATION, AND RESISTANCE AGAINST MICROORGANISMS AMONG THE HEN GENOTYPES EXIST?*}

\author{
Adam Kraus $^{1 \star}$, Lukáš Zita ${ }^{1}$, Ondřej Krunt ${ }^{1}$, Darina Chodová1, Monika Okrouhlá ${ }^{1}$, Józefa Krawczyk² \\ ${ }^{1}$ Department of Animal Science, Faculty of Agrobiology, Food and Natural Resources, \\ Czech University of Life Sciences Prague, Kamýcká 129, 16500 Prague - Suchdol, Czech Republic \\ ${ }^{2}$ Department of Poultry Breeding, National Research Institute of Animal Production, \\ 32-083 Balice n. Kraków, Poland \\ •Corresponding author: krausa@af.czu.cz
}

\begin{abstract}
The aim of this study was to evaluate and compare the impact of genotype and storage conditions (temperature and time) on microbiological contamination and eggshell quality. There were four genotypes of laying hens used, Czech Golden Spotted (CGS), Greenleg Partridge (GP), White Leghorn (WL) and commercial hybrid (CH) hens were included. After collection, the eggs were divided equally into five groups according to the storage time $\left(0,14,28\right.$ days) and temperature $\left(5\right.$ and $\left.20^{\circ} \mathrm{C}\right)$. The microbiological analysis included counting of colonies forming units (CFU) of Escherichia coli (EC), Enterococcus (ENT) and total number of microorganisms (TNM) on eggshell surface, eggshell membranes and in thin albumen. The analysis of eggshell quality included the determination of eggshell proportion (SP), thickness (ST), strength (SST), index (SI) and surface (SS). Moreover, egg weight (EW) and egg weight loss (EWL) were determined. The significant effect of genotype was found in contamination of eggshell by EC, ENT and TNM, eggshell membranes by TNM and albumen by EC (all P $\leq$ 0.05). The significantly lowest contamination of eggshell from EC was in eggs from the WL hens (4.42 log CFU/ eggshell), while from ENT was in eggs from the CGS hens (1.22 log CFU/eggshell) and from the WL hens (1.40 log CFU/eggshell). The lowest incidence of TNM was also detected in eggs from the WL hens (5.03 log CFU/eggshell). Statistically the lowest contamination of eggshell membranes by TNM was found in eggs from the WL (0.12 log CFU/eggshell membranes) and CH hens (0.15 log CFU/eggshell membranes). Regarding the effect of genotype, the GP (not detected) and WL (not detected) hens had eggs with statistically the lowest occurrence of EC bacteria in albumen. Regarding the EW and eggshell quality, all the parameters were significantly affected by the genotype $(\mathrm{P} \leq \mathbf{0 . 0 0 0 1})$. Also EWL was significantly $(\mathrm{P} \leq \mathbf{0 . 0 5})$ affected by genotype (after 14,21 and 28 days of storage). There were found to be significant differences of microbial contamination of egg surface among observed hen genotypes. The penetration of selected microorganisms was also significant in contamination of eggshell membranes by TNM and in contamination of albumen by EC.
\end{abstract}

Key words: egg, genotype, microorganisms, storage temperature, storage time

In recent years, food safety has become a more discussed topic and greater emphasis is being put on its improvement, modernization and strengthening (Elmi, 2004). Food safety is a tool that prevents the spreading of health dangerous food to consumers causing foodborne illnesses. One of the main components of food safety are storage conditions (FAO, 2003). Moreover, refrigeration during both transport and storage, is an essential factor specifically for food safety of eggs, respectively of egg products. Special care and handling is necessary to avoid unfavourable contamination (Zaheer, 2015). Eggs belong to worldwide spread food, which is valued especially for good nutritional value. Hens' eggs represent a great source of high quality proteins, essential fatty acids (Iannotti et al., 2014), fat-soluble vitamins (A, D, E, and $\mathrm{K})$, water-soluble vitamins (B) and minerals, such as cal- cium, iron, magnesium, phosphorus, selenium, sodium and zinc (Zaheer, 2015).

In general, several numbers of internal and external factors may affect different aspects of egg quality (Roberts, 2004). Kraus et al. (2019) consider age, genotype and nutrition as the most influential factors. Englmaierová et al. (2014) describe the level of microbiological contamination as an important factor, which influences overall egg quality. Storage conditions including method, time and temperature, play a considerable role as well (Svobodová and Tůmová, 2014; Brodacki et al., 2019). A number of authors, such as Jones et al. (2004), Vlčková et al. (2018) and Krunt et al. (2021) studied the effect of storage conditions on microbiological contamination. Jones et al. (2004) determined the effect of genotype and storage time, Vlčková et al. (2018) the effect of storage

\footnotetext{
*Source of research financing: "S" grant of Ministry of Education, Youth and Sports of the Czech Republic and grant project no. SV21-6-
} 21320 
time, housing system and age of hens and Krunt et al. (2021) the effect of storage time, storage temperature and genotype. As mentioned above, some environmental factors have an impact on the contamination of eggs, so the avians have throughout the years adapted and developed several mechanisms, such as behavioural, chemical and physiological, to fight with the infection. However, the microbial contamination of eggs begins with the contact of an egg with the floor surface, nevertheless certain bacteria, such as Enterococcus spp., occur in the digestive tract (D'Alba and Shawkey, 2015).

Microbiological contamination represents an "invisible" threat to egg quality (Rodríguez-Navarro et al., 2013). As an indicator of egg safety, Enterobacteriaceae and total bacteria populations are commonly used (Moyle et al., 2016). These gram-negative bacteria are mostly detected inside of contaminated eggs, because of their higher resistance to antimicrobial proteins, such as lysozyme (D'Alba and Shawkey, 2015). Bacteria are able to penetrate from the egg surface, through the eggshell membranes into the internal egg content. Due to this fact, it is essential to focus on bacterial count not only on the egg surface, but also in the internal parts of eggs (Moyle et al., 2016). The count of bacteria on the egg surface define the probability of penetration through the eggshell into the internal content (D'Alba and Shawkey, 2015). The eggshell quality is important not only because it protects the egg content (Vlčková et al., 2018), but also in terms of the relationship with a microbiological contamination of egg internal parts (Zaheer, 2015). Furthermore, the eggshell quality is also important for producers from the economic point of view. The mechanical damage (cracks) and uncommon changes (especially in egg shape) are unfavourable (Hernandez et al., 2005).

Nowadays, there is still greater emphasis put on high production, especially in developed countries (Hanusová et al., 2017), while at the same time on the choice of a suitable housing system regarding better welfare and health condition of animals (Dikmen et al., 2016). For housing of native breeds, alternative housing systems are more suitable in comparison with cage systems (Zita et al., 2018; Kraus et al., 2021). Intensive breeding causes the loss of many valuable genes, so it is essential to keep the breeding of native breeds (Hanusová et al., 2017). Czech Golden Spotted (Anderle et al., 2014) and Greenleg Partridge hens (Krawczyk, 2009) are native breeds of the Czech Republic and Poland respectively, while White Leghorn hens belong among the most important original breeds (FAO, 2007).

The aim of this study was to evaluate and compare the impact of genotype and storage conditions, specifically temperature and time, on microbiological contamination and eggshell quality.

\section{Material and methods}

The Ethics Committee of the Central Commission for Animal Welfare at the Ministry of Agriculture of the
Czech Republic and the Ethics Committee of the Czech University of Life Sciences Prague approved this study with animals (approval document no. 07/2020).

\section{Animals and management}

There were four genotypes of laying hens used, three native breeds and commercial hybrid. Specifically, Czech Golden Spotted (CGS), Greenleg Partridge (GP), White Leghorn (WL) and commercial hybrid $(\mathrm{CH})$ hens were included. The Hy-Line brown egg-laying hybrid was used as a representative of the group labelled as commercial hybrid.

CGS hens belong to gene reserves of the Czech Republic (Anderle et al., 2014). The main exterior characteristics include typical lightweight type (body weight of adult hens can reach up to $2.0 \mathrm{~kg}$ ), medium body frame, cylindrical body shape, leaf type of comb, red ears, rich tail, medium high stance, white skin and slate colored shanks (Zita et al., 2018). The CGS hens lay eggs with a creamy coloured eggshell and average egg weight ranges from 57 to $58 \mathrm{~g}$ (Anderle et al., 2014). Zita et al. (2018) state that average egg weight is lower, specifically from 51.56 to $53.33 \mathrm{~g}$ depending on the housing system. They also calculated hen-day egg production, which reached $24.49 \%$ in cage housed hens and $30.85 \%$ in litter housed hens. These findings can be supported by results from Kraus et al. (2021), who concluded that CGS hens are more suitable for breeding in non-cage housing systems. GP is the oldest native Polish breed of hens. This breed is characterised by its lower body weight $(1.7-1.8 \mathrm{~kg})$, green legs, and grey, partridge-like plumage. GP hens lay eggs with a creamy coloured eggshell and with high yolk proportion (Batkowska and Brodacki, 2017). According to Krawczyk (2009), average egg weight of eggs from GP hens varies between 54.1 and $57.3 \mathrm{~g}$ depending on the housing system. Average hen-day egg production of this breed reaches from $56.5 \%$ to $60.7 \%$ (Krawczyk et al., 2011). WL hens represent a breed, which lays eggs with a white eggshell (Hanusová et al., 2015) and with egg weight between 54.27 and $62.92 \mathrm{~g}$. Hen-day egg production may vary from 70 to $89 \%$ (Pohle and Cheng, 2009). According to Jones et al. (2001), hen-day egg production of WL hens is lower, specifically between 56.88 and $73.38 \%$. Another characteristic of this breed is its body constitution, body weight typically reaches values from 1.42 to $1.71 \mathrm{~kg}$. All these parameters depend on many factors, mainly on hen age or housing system (Pohle and Cheng, 2009). CH hens were represented by HyLine brown hens in this study. These hens lay eggs with a brown eggshell colour with egg weight between 57.3 and 66.7 g. Hen-day egg production can reach up to 95 $96 \%$ at peak. Body weight ranges from 1.85 to $2.04 \mathrm{~kg}$ in adult hens (Hy-Line, 2021).

The number of hens kept for the purpose of this study was adjusted to match the number of CGS hens, because there is a very limited amount of officially recognized hens of this breed. Therefore, 50 hens of each genotype were used. The hens of each genotype were divided into 
half and kept separately because of the replication of the results. Hens were kept in an external experimental centre in the Department of Animal Science of the Faculty of Agrobiology, Food and Natural Resources (Czech University of Life Sciences Prague). All hens were housed on litter under the same housing conditions including the microclimate conditions, lighting regime, composition of feed mixture and access to feed and water. The requirements on floor area in litter housing systems (maximum density of 9 hens per $\mathrm{m}^{2}$ ), which are set by the European Commission Directive 1999/74/EC, were met. The temperature in the halls was kept between 18 and $20^{\circ} \mathrm{C}$ and humidity between 50 and $60 \%$. In terms of the lighting regime, natural conditions were used. Feed mixture contained $16.4 \%$ of crude protein and $11.42 \mathrm{MJ}$ of metabolizable energy. The access to both feed and water was ad libitum.

\section{Samples}

A total of 200 eggs (50 from each genotype) were used for a microbiological analysis, while 800 eggs (200 from each genotype) were used for the eggshell quality analysis. The hens were 44 weeks old when the collection of eggs was carried out. The eggs were collected using sterile plastic gloves and then placed into sterile plastic boxes to avoid any undesirable contamination. All eggs fell into the weight category $\mathrm{M}$, which ranges from 53 to $63 \mathrm{~g}$. The collection was carried out for the duration of the whole week to achieve the required number of eggs for the analysis. After the collection, eggs were equally divided into five groups. The first group was analysed immediately as a control group marked as a day 0 group (fresh eggs). The second and third groups were both stored for 14 days, the second at a temperature of $5^{\circ} \mathrm{C}$ and the third at a temperature of $20^{\circ} \mathrm{C}$. The last two groups were stored for 28 days, which is the official minimal shelf life of eggs. The last two groups were also divided according to the temperature, as the previous ones.

The eggs were analysed immediately after the collection (day 0 group) or were immediately put into the specific storage conditions for a specific group. The eggs were stored in the fridge (with air access), where the temperature was kept at $5^{\circ} \mathrm{C}$ and relative humidity at 50-60\% and in a room with a thermostat, where the temperature was kept at $20^{\circ} \mathrm{C}$ and relative humidity at $50-60 \%$ respectively. Digital thermometers Emos E8860 were used for the monitoring of the required conditions. The temperature and relative humidity were controlled and recorded twice a day. All laboratory analyses were carried out in the laboratory of the Department of Animal Science of the Faculty of Agrobiology, Food and Natural Resources of the Czech University of Life Sciences Prague.

\section{Microbiological analysis}

The microbiological analysis was made according to Krunt et al. (2021) and included the counting of colonies forming units (CFU) of Escherichia coli (EC), Enterococcus (ENT) and a total number of microorganisms (TNM). The determination of microbiological con- tamination was made from the eggshell surface, eggshell membranes and thin albumen. The analysis of microbial contamination consisted of a number of steps. For the determination of eggshell contamination, eggs were placed into sterile plastic bags (each egg was placed into a separate bag) with a $10 \mathrm{ml}$ solution of saline peptone ( $9 \mathrm{~g}$ of sodium chloride, $1 \mathrm{~g}$ of peptone, and $1000 \mathrm{ml}$ of distilled water; Sigma-Aldrich, Saint Louis, USA). All eggs were thoroughly rinsed for 120 seconds in the plastic bags. After this procedure, the eggs were removed from the plastic bags and then a dilution series for each egg was made by adding $1 \mathrm{ml}$ of the solution $\left(10^{0}, 10^{-1}, 10^{-2}, 10^{-3}, 10^{-4}\right.$ and $\left.10^{-5}\right)$. The dilution $10^{\circ}$ was prepared directly from the plastic bag. The determination of the microbiological contamination of the eggshell membranes and albumen was subjected to the same procedure as the determination of the eggshell contamination. Prior to the eggshell membranes and albumen samples preparation, the eggshell surface was disinfected by rinsing in ethanol so the subsequent removal of the eggshell membranes, respectively of the albumen was sterile. The dilution series was prepared the same way as in the determination of eggshell contamination. However, the difference was in the dilution $10^{\circ}$ that contained directly eggshell membrane, respectively albumen. When the dilution series was prepared, $1 \mathrm{ml}$ of the solution from each dilution for EC and ENT and $0.1 \mathrm{ml}$ for TNM, was put into a Petri dish with a specific agar (or the agar was added after the sample application in the case of TNM). Each sample was analysed in duplicate. Regarding the agar methods, standard methods were used. The Mac-Conkey agar was used for the count of EC, Slanetz Bartley agar was used for the count of ENT and the Standard Plate Count agar was used for the count of TNM (all Oxoid ${ }^{\mathrm{TM}}$, Thermo Scientific, Tewksburg, USA). The samples for the determination of EC (Mac-Conkey agar) and ENT (Slanetz Bartley agar) were incubated at $37^{\circ} \mathrm{C}$ for $48 \mathrm{~h}$ in an incubator. The samples for the determination of TNM (Standard Plate Count Agar) were incubated at $30^{\circ} \mathrm{C}$ for $120 \mathrm{~h}$ in an incubator (Memmert INE 500). After the incubation, the count of typical CFU was made for each Petri dish. The final contamination of individual parts was calculated by the standard plate count method formula.

\section{Eggshell quality analysis}

The analysis of eggshell quality included the determination of eggshell proportion (SP), thickness (ST), strength (SST), index (SI) and surface (SS). Even though egg weight (EW) does not belong to the eggshell parameters, it was determined. Furthermore, egg weight loss (EWL) was determined as well.

The EW and the eggshell weight (SW), which is required for the calculation of ESP, were measured by laboratory scale Ohaus (Model: Traveler TA502, Parsippany, NJ 07054, USA) with $0.01 \mathrm{~g}$ precision. The ST was determined by a digital micrometer (Digimatic Outside Micrometer, Mitutoyo Corporation, Japan) with $0.001 \mathrm{~mm}$ precision. The ST was measured without eggshell mem- 
branes at the center of the eggshell, the measurements of each eggshell were made in duplicate. The SST was assessed by an Instron device (Instron Universal Testing Machine; model 3342; Instron Ltd., US), which calculates the necessary force (in $\mathrm{N} / \mathrm{cm}^{2}$ ) for eggshell breakage. Furthermore, SS was calculated by the formula SS $=4.68 \times \mathrm{EW}^{2 / 3}\left[\mathrm{~cm}^{2}\right]$, where $\mathrm{EW}$ is the egg weight in $\mathrm{g}$ (Ahmed et al., 2005). For the determination of SI, the formula $\mathrm{SI}=(\mathrm{SW} / \mathrm{SS}) \times 100\left[\mathrm{~g} / 100 \mathrm{~cm}^{2}\right]$, where $\mathrm{SW}$ is eggshell weight in $\mathrm{g}$ and $\mathrm{SS}$ is eggshell surface in $\mathrm{cm}^{2}$ (Ahmed et al., 2005).

\section{Statistical analysis}

The data were analysed by statistical software SAS 9.4 (SAS Institute Inc., Cary, NC, 2012). The data were tested for normality with univariate plot normal procedure of SAS and subsequently subjected to a three-way ANOVA in a 4 (genotype: CGS, GP, CH, WL hens) $\times 3$ (storage time: $0,14,28$ days) $\times 3$ (storage temperature: fresh, $5^{\circ} \mathrm{C}, 20^{\circ} \mathrm{C}$ ) factorial arrangement of treatments using the Tukey test by the PROC MIX procedure of SAS. The value of $\mathrm{P} \leq 0.05$ was considered statistically significant. The results in the tables show the average values of each treatment and the standard error of the mean (SEM).

\section{Results}

The results of this study are presented in following tables. The microbiological contamination of the eggshell surface is described in Table 1, while the microbiological contamination of eggshell membranes in Table 2. The microbiological contamination of albumen is presented in Table 3. EW and eggshell quality parameters are shown in Table 4. EWL data are shown in Table 5. All statistically significant interactions are discussed in detail in the text, but not all of them are described in the tables.

Table 1. Effect of genotype, storage time and temperature on microbial contamination of the eggshell (CFU/eggshell)

\begin{tabular}{|c|c|c|c|c|c|}
\hline $\mathrm{G}$ & STI (days) & $\operatorname{STE}\left({ }^{\circ} \mathrm{C}\right)$ & $\mathrm{EC}$ & ENT & TNM \\
\hline 1 & 2 & 3 & 4 & 5 & 6 \\
\hline CGS & & & $5.31 \mathrm{a}$ & $1.22 \mathrm{~b}$ & $5.77 \mathrm{a}$ \\
\hline GP & & & $5.11 \mathrm{a}$ & $1.86 \mathrm{ab}$ & $5.24 \mathrm{ab}$ \\
\hline $\mathrm{CH}$ & & & $5.21 \mathrm{a}$ & $2.28 \mathrm{a}$ & $5.81 \mathrm{a}$ \\
\hline \multirow[t]{9}{*}{ WL } & & & $4.42 \mathrm{~b}$ & $1.40 \mathrm{~b}$ & $5.03 \mathrm{~b}$ \\
\hline & 0 & & 5.59 & $2.10 \mathrm{a}$ & 6.02 \\
\hline & 14 & & 4.74 & $1.92 \mathrm{a}$ & 5.46 \\
\hline & 28 & & 4.93 & $1.16 \mathrm{~b}$ & 5.10 \\
\hline & & fresh & 5.59 & $2.10 \mathrm{a}$ & $6.02 \mathrm{a}$ \\
\hline & & 5 & 4.89 & $1.97 \mathrm{a}$ & $5.56 \mathrm{ab}$ \\
\hline & & 20 & 4.78 & $1.12 \mathrm{~b}$ & $5.00 \mathrm{~b}$ \\
\hline & 0 & fresh & 5.88 & 2.01 & 6.03 \\
\hline & 14 & 5 & 5.21 & 1.94 & 5.38 \\
\hline \multirow{5}{*}{ CGS } & 14 & 20 & 4.97 & 0.41 & 6.08 \\
\hline & 28 & 5 & 5.07 & 1.22 & 5.85 \\
\hline & 28 & 20 & 5.04 & 0 & 5.34 \\
\hline & 0 & fresh & 5.51 & 1.85 & 5.75 \\
\hline & 14 & 5 & 5.14 & 3.69 & 5.57 \\
\hline \multirow[t]{5}{*}{ GP } & 14 & 20 & 4.67 & 1.15 & 5.72 \\
\hline & 28 & 5 & 5.18 & 2.62 & 6.22 \\
\hline & 28 & 20 & 4.70 & 0 & 2.60 \\
\hline & 0 & fresh & 5.95 & 3.37 & 6.56 \\
\hline & 14 & 5 & 4.78 & 3.37 & 5.49 \\
\hline \multirow[t]{5}{*}{$\mathrm{CH}$} & 14 & 20 & 4.70 & 1.91 & 5.69 \\
\hline & 28 & 5 & 5.06 & 2.98 & 5.87 \\
\hline & & 20 & 5.56 & 2.09 & 5.45 \\
\hline & 0 & fresh & 4.82 & 1.42 & 5.91 \\
\hline & 14 & 5 & 4.61 & 1.41 & 4.95 \\
\hline \multirow[t]{3}{*}{ WL } & 14 & 20 & 3.87 & 1.89 & 4.78 \\
\hline & 28 & 5 & 4.03 & 0.81 & 5.17 \\
\hline & 20 & 20 & 4.77 & 1.48 & 4.30 \\
\hline
\end{tabular}


Table $1-$ contd

\begin{tabular}{|c|c|c|c|c|c|}
\hline 1 & 2 & 3 & 4 & 5 & 6 \\
\hline \multicolumn{6}{|l|}{ P-value } \\
\hline G & & & $\leq 0.05$ & $\leq 0.05$ & $\leq 0.05$ \\
\hline STI & & & 0.2720 & $\leq 0.05$ & 0.1422 \\
\hline STE & & & 0.5316 & $\leq 0.05$ & $\leq 0.05$ \\
\hline $\mathrm{G} \times \mathrm{STI}$ & & & 0.5553 & 0.8907 & 0.2152 \\
\hline $\mathrm{G} \times \mathrm{STE}$ & & & 0.5059 & $\leq 0.05$ & $\leq 0.05$ \\
\hline $\mathrm{STI} \times \mathrm{STE}$ & & & 0.0910 & 0.3090 & $\leq 0.05$ \\
\hline $\mathrm{G} \times \mathrm{STI} \times \mathrm{STE}$ & & & 0.3943 & 0.5833 & 0.0780 \\
\hline SEM & & & 0.0885 & 0.1637 & 0.1332 \\
\hline
\end{tabular}

CFU - colony forming units, G - genotype; STI - storage time; STE - storage temperature; EC - Escherichia coli, ENT - Enterococcus, TNM - total number of microorganisms, CGS - Czech Golden Spotted hen; GP - Greenleg Partridge; CH - commercial hybrid; WL - White Leghorn; SEM - standard error of mean; Values with significance of $\mathrm{P} \leq 0.05$ were considered as significant.

Table 2. Effect of genotype, storage time and temperature on microbial contamination of the eggshell membranes (CFU/eggshell membranes)

\begin{tabular}{|c|c|c|c|c|c|}
\hline G & STI (days) & $\operatorname{STE}\left({ }^{\circ} \mathrm{C}\right)$ & $\mathrm{EC}$ & ENT & TNM \\
\hline 1 & 2 & 3 & 4 & 5 & 6 \\
\hline CGS & & & 0.32 & 0 & $0.82 \mathrm{a}$ \\
\hline GP & & & 0.27 & 0 & $0.61 \mathrm{ab}$ \\
\hline $\mathrm{CH}$ & & & 0 & 0 & $0.15 \mathrm{~b}$ \\
\hline \multirow[t]{9}{*}{ WL } & & & 0.26 & 0.11 & $0.12 b$ \\
\hline & 0 & & $0 \mathrm{~b}$ & 0 & $0.28 \mathrm{~b}$ \\
\hline & 14 & & $0.51 \mathrm{a}$ & 0 & $0.76 \mathrm{a}$ \\
\hline & 28 & & $0.07 \mathrm{~b}$ & 0.07 & $0.23 \mathrm{~b}$ \\
\hline & & fresh & 0 & 0 & 0.28 \\
\hline & & 5 & 0.19 & 0 & 0.51 \\
\hline & & 20 & 0.39 & 0.07 & 0.49 \\
\hline & 0 & fresh & 0 & 0 & 0.36 \\
\hline & 4 & 5 & 0.57 & 0 & 1.46 \\
\hline \multirow{5}{*}{ CGS } & 14 & 20 & 1.23 & 0 & 1.75 \\
\hline & 28 & 5 & 0 & 0 & 0.70 \\
\hline & 28 & 20 & 0 & 0 & 0.47 \\
\hline & 0 & fresh & 0 & 0 & 0.26 \\
\hline & 14 & 5 & 0.95 & 0 & 1.62 \\
\hline \multirow[t]{5}{*}{ GP } & 14 & 20 & 0.57 & 0 & 1.20 \\
\hline & 20 & 5 & 0 & 0 & 0 \\
\hline & 28 & 20 & 0 & 0 & 0.23 \\
\hline & 0 & fresh & 0 & 0 & 0 \\
\hline & 14 & 5 & 0 & 0 & 0.13 \\
\hline \multirow[t]{5}{*}{$\mathrm{CH}$} & 14 & 20 & 0 & 0 & 0.13 \\
\hline & 28 & 5 & 0 & 0 & 0.48 \\
\hline & 20 & 20 & 0 & 0 & 0 \\
\hline & 0 & fresh & 0 & 0 & 0.48 \\
\hline & 14 & 5 & 0 & 0 & 0 \\
\hline \multirow[t]{3}{*}{ WL } & 14 & 20 & 0.73 & 0 & 0.13 \\
\hline & 28 & 5 & 0 & 0 & 0 \\
\hline & 20 & 20 & 0.57 & 0.57 & 0 \\
\hline
\end{tabular}




\begin{tabular}{l|c|c|c|c}
\multicolumn{1}{c}{ Table 2-contd. } \\
\hline \multicolumn{1}{c|}{1} & 3 & 4 & 5 & 6 \\
\hline P-value & 2 & & & \\
G & & 0.5124 & 0.4816 & $\leq 0.05$ \\
STI & $\leq 0.05$ & 0.2473 & $\leq 0.05$ \\
STE & 0.2732 & 0.2473 & 0.9109 \\
G $\times$ STI & & 0.1853 & 0.2629 & $\leq 0.05$ \\
G $\times$ STE & & 0.3667 & 0.2629 & 0.8600 \\
STI $\times$ STE & & 0.7563 & 0.2473 & 0.5931 \\
G $\times$ STI $\times$ STE & & 0.7720 & 0.2629 & 0.5334 \\
SEM & & 0.0794 & 0.0265 & 0.0936 \\
\hline
\end{tabular}

CFU - colony forming units, G - genotype; STI - storage time; STE - storage temperature; EC - Escherichia coli, ENT - Enterococcus, TNM - total number of microorganisms, CGS - Czech Golden Spotted hen; GP - Greenleg Partridge; CH - commercial hybrid; WL - White Leghorn; SEM - standard error of mean; Values with significance of $\mathrm{P} \leq 0.05$ were considered as significant.

Table 3. Effect of genotype, storage time and temperature on microbial contamination of the albumen (CFU/albumen)

\begin{tabular}{|c|c|c|c|c|c|}
\hline G & STI (days) & $\operatorname{STE}\left({ }^{\circ} \mathrm{C}\right)$ & $\mathrm{EC}$ & ENT & TNM \\
\hline 1 & 2 & 3 & 4 & 5 & 6 \\
\hline CGS & & & $0.52 \mathrm{a}$ & 0 & 0.21 \\
\hline GP & & & $0 \mathrm{~b}$ & 0 & 0.16 \\
\hline $\mathrm{CH}$ & & & $0.11 \mathrm{ab}$ & 0 & 0.17 \\
\hline \multirow[t]{9}{*}{ WL } & & & $0 \mathrm{~b}$ & 0 & 0.21 \\
\hline & 0 & & 0 & 0 & 0.29 \\
\hline & 14 & & 0.30 & 0 & 0.10 \\
\hline & 28 & & 0.14 & 0 & 0.20 \\
\hline & & fresh & 0 & 0 & 0.29 \\
\hline & & 5 & 0.30 & 0 & 0.22 \\
\hline & & 20 & 0.14 & 0 & 0.09 \\
\hline & 0 & fresh & 0 & 0 & 0.42 \\
\hline & 14 & 5 & 1.23 & 0 & 0 \\
\hline \multirow{5}{*}{ CGS } & 14 & 20 & 0.57 & 0 & 0 \\
\hline & 28 & 5 & 1.13 & 0 & 0.45 \\
\hline & 20 & 20 & 0 & 0 & 0 \\
\hline & 0 & fresh & 0 & 0 & 0.14 \\
\hline & 14 & 5 & 0 & 0 & 0 \\
\hline \multirow[t]{5}{*}{ GP } & 14 & 20 & 0 & 0 & 0 \\
\hline & 28 & 5 & 0 & 0 & 0 \\
\hline & 20 & 20 & 0 & 0 & 0.70 \\
\hline & 0 & fresh & 0 & 0 & 0 \\
\hline & 14 & 5 & 0 & 0 & 0.83 \\
\hline \multirow[t]{5}{*}{$\mathrm{CH}$} & 17 & 20 & 0.57 & 0 & 0 \\
\hline & 28 & 5 & 0 & 0 & 0 \\
\hline & 20 & 20 & 0 & 0 & 0 \\
\hline & 0 & fresh & 0 & 0 & 0.60 \\
\hline & 14 & 5 & 0 & 0 & 0 \\
\hline \multirow[t]{3}{*}{ WL } & 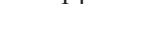 & 20 & 0 & 0 & 0 \\
\hline & 28 & 5 & 0 & 0 & 0.47 \\
\hline & 20 & 20 & 0 & 0 & 0 \\
\hline
\end{tabular}




\begin{tabular}{|c|c|c|c|c|c|}
\hline 1 & 2 & 3 & 4 & 5 & 6 \\
\hline \multicolumn{6}{|l|}{ P-value } \\
\hline G & & & $\leq 0.05$ & - & 0.9378 \\
\hline STI & & & 0.2199 & - & 0.5032 \\
\hline STE & & & 0.2199 & - & 0.3735 \\
\hline $\mathrm{G} \times \mathrm{STI}$ & & & 0.6687 & - & 0.2603 \\
\hline $\mathrm{G} \times \mathrm{STE}$ & & & $\leq 0.05$ & - & 0.2977 \\
\hline $\mathrm{STI} \times \mathrm{STE}$ & & & 0.3031 & - & 0.6157 \\
\hline $\mathrm{G} \times \mathrm{STI} \times \mathrm{STE}$ & & & 0.7759 & - & 0.2411 \\
\hline SEM & & & 0.644 & - & 0.0634 \\
\hline
\end{tabular}

CFU - colony forming units, G - genotype; STI - storage time; STE - storage temperature; EC - Escherichia coli, ENT - Enterococcus, TNM - total number of microorganisms, CGS - Czech Golden Spotted hen; GP - Greenleg Partridge; CH - commercial hybrid; WL - White Leghorn; SEM - standard error of mean; Values with significance of $\mathrm{P} \leq 0.05$ were considered as significant.

Table 4. Effect of genotype, storage time and temperature on egg weight and eggshell quality parameters

\begin{tabular}{|c|c|c|c|c|c|c|c|c|}
\hline G & STI (days) & $\begin{array}{l}\text { STE } \\
\left({ }^{\circ} \mathrm{C}\right)\end{array}$ & $\begin{array}{r}\text { EW } \\
(\mathrm{g})\end{array}$ & $\begin{array}{l}\text { SP } \\
(\%)\end{array}$ & $\begin{array}{c}\mathrm{ST} \\
(\mathrm{mm})\end{array}$ & $\operatorname{SST}\left(\mathrm{N} / \mathrm{cm}^{2}\right)$ & $\begin{array}{c}\text { SI } \\
\left(\mathrm{g} / 100 \mathrm{~cm}^{2}\right)\end{array}$ & $\begin{array}{c}\mathrm{SS} \\
\left(\mathrm{cm}^{2}\right)\end{array}$ \\
\hline 1 & 2 & 3 & 4 & 5 & 6 & 7 & 8 & 9 \\
\hline CGS & & & $56.42 \mathrm{~b}$ & $9.45 \mathrm{ab}$ & $0.306 \mathrm{ab}$ & $42.70 \mathrm{a}$ & $7.74 \mathrm{ab}$ & $68.83 \mathrm{ab}$ \\
\hline GP & & & $55.77 \mathrm{~b}$ & $8.90 \mathrm{c}$ & $0.292 \mathrm{~b}$ & $34.60 \mathrm{c}$ & $7.26 \mathrm{c}$ & $68.30 \mathrm{~b}$ \\
\hline $\mathrm{CH}$ & & & $57.97 \mathrm{a}$ & $9.25 \mathrm{~b}$ & $0.307 \mathrm{ab}$ & $38.53 \mathrm{~b}$ & $7.65 \mathrm{~b}$ & $70.08 \mathrm{a}$ \\
\hline \multirow[t]{8}{*}{ WL } & & & $55.85 \mathrm{~b}$ & $9.72 \mathrm{a}$ & $0.314 \mathrm{a}$ & $38.34 \mathrm{~b}$ & $7.94 \mathrm{a}$ & $68.36 \mathrm{~b}$ \\
\hline & 0 & & $57.96 \mathrm{a}$ & $9.19 \mathrm{~b}$ & $0.309 \mathrm{a}$ & 39.09 & $7.59 \mathrm{~b}$ & $70.08 \mathrm{a}$ \\
\hline & 14 & & $56.52 \mathrm{~b}$ & $9.22 \mathrm{~b}$ & $0.297 \mathrm{~b}$ & 38.05 & $7.56 \mathrm{~b}$ & $68.90 \mathrm{ab}$ \\
\hline & 28 & & $55.76 \mathrm{c}$ & $9.51 \mathrm{a}$ & $0.311 \mathrm{a}$ & 38.65 & $7.76 \mathrm{a}$ & $68.28 \mathrm{~b}$ \\
\hline & & fresh & $57.96 \mathrm{a}$ & $9.19 \mathrm{~b}$ & $0.309 \mathrm{a}$ & 39.09 & $7.59 \mathrm{~b}$ & $70.08 \mathrm{a}$ \\
\hline & & 5 & $56.81 \mathrm{~b}$ & $9.23 \mathrm{~b}$ & $0.310 \mathrm{a}$ & 38.19 & $7.78 \mathrm{a}$ & $69.14 \mathrm{~b}$ \\
\hline & & 20 & $55.47 \mathrm{c}$ & $9.50 \mathrm{a}$ & $0.297 \mathrm{~b}$ & 38.51 & $7.74 \mathrm{a}$ & $68.05 \mathrm{c}$ \\
\hline & 0 & fresh & 57.67 & 9.32 & 0.311 & 43.42 & 7.69 & 69.84 \\
\hline \multirow{6}{*}{ CGS } & 14 & 5 & 56.80 & 9.26 & 0.306 & 41.39 & 7.60 & 69.13 \\
\hline & 17 & 20 & 55.88 & 9.58 & 0.292 & 42.76 & 7.82 & 68.39 \\
\hline & 28 & 5 & 57.35 & 9.23 & 0.309 & 42.73 & 7.61 & 69.58 \\
\hline & 20 & 20 & 54.43 & 9.87 & 0.313 & 43.20 & 7.99 & 67.20 \\
\hline & 0 & fresh & 56.83 & 8.79 & 0.296 & 34.79 & 7.22 & 69.16 \\
\hline & 14 & 5 & 56.86 & 8.79 & 0.300 & 34.36 & 7.20 & 69.19 \\
\hline \multirow[t]{5}{*}{ GP } & tT & 20 & 55.38 & 8.85 & 0.285 & 33.40 & 7.21 & 67.98 \\
\hline & 28 & 5 & 55.99 & 8.89 & 0.292 & 35.74 & 7.27 & 68.48 \\
\hline & 20 & 20 & 53.79 & 9.21 & 0.287 & 34.73 & 7.43 & 66.68 \\
\hline & 0 & fresh & 60.19 & 9.03 & 0.309 & 41.09 & 7.56 & 71.87 \\
\hline & & 5 & 58.98 & 8.75 & 0.300 & 35.68 & 7.27 & 70.91 \\
\hline \multirow[t]{5}{*}{$\mathrm{CH}$} & 14 & 20 & 57.68 & 9.03 & 0.277 & 37.38 & 7.46 & 69.85 \\
\hline & 28 & 5 & 58.15 & 9.25 & 0.319 & 37.55 & 7.66 & 70.22 \\
\hline & & 20 & 54.86 & 10.16 & 0.330 & 40.06 & 8.25 & 67.55 \\
\hline & 0 & fresh & 57.17 & 9.60 & 0.319 & 37.08 & 7.90 & 69.43 \\
\hline & 14 & 5 & 54.36 & 9.87 & 0.326 & 39.52 & 7.98 & 67.12 \\
\hline \multirow[t]{3}{*}{ WL } & 14 & 20 & 56.22 & 9.68 & 0.292 & 39.94 & 7.92 & 68.66 \\
\hline & 28 & 5 & 56.01 & 9.82 & 0.331 & 38.55 & 8.02 & 68.48 \\
\hline & 20 & 20 & 55.50 & 9.65 & 0.306 & 36.60 & 7.86 & 68.07 \\
\hline
\end{tabular}


Table 4 - contd.

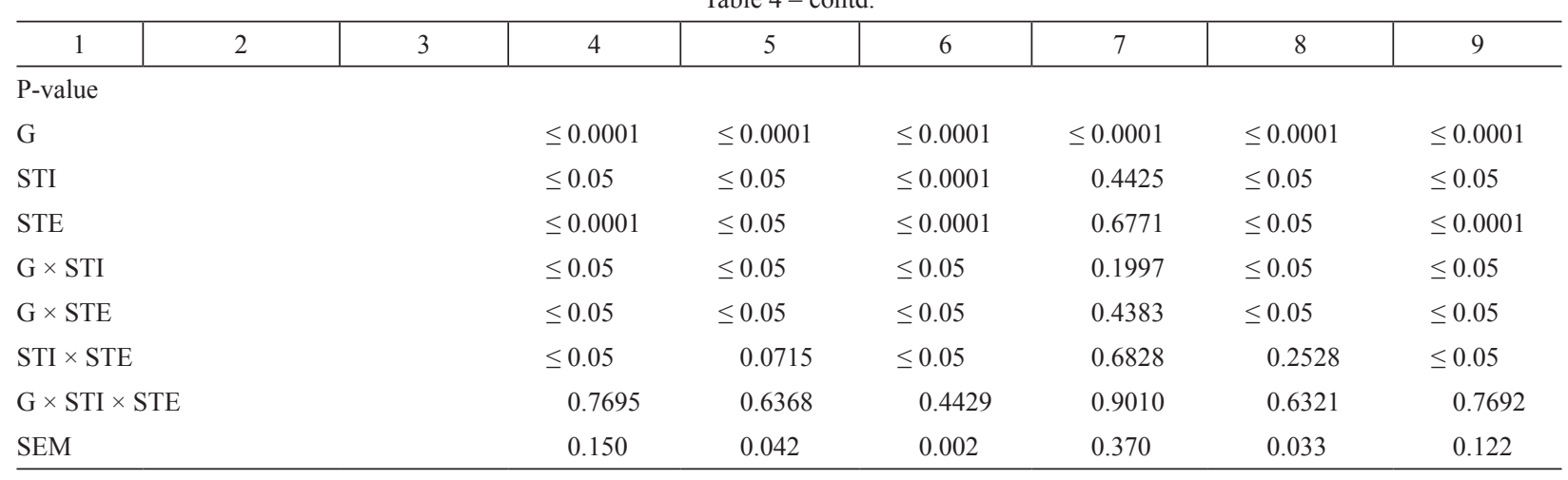

G - genotype; STI - storage time; STE - storage temperature; EW - egg weight; ESW - eggshell weight; SP - eggshell proportion; ST - eggshell thickness; SST - eggshell strength; SI - eggshell index; SS - eggshell surface; CGS - Czech Golden Spotted hen; GP - Greenleg Partridge; CH - commercial hybrid; WL - White Leghorn; SEM - standard error of mean; Values with significance of $\mathrm{P} \leq 0.05$ were considered as significant.

Table 5. Effect of genotype, storage time and temperature on egg weight loss after 7, 14, 21 and 28 days from the initial EW

\begin{tabular}{|c|c|c|c|c|c|c|}
\hline \multirow{2}{*}{ G } & \multirow{2}{*}{$\begin{array}{c}\text { STI } \\
\text { (days) }\end{array}$} & \multirow{2}{*}{$\begin{array}{l}\text { STE } \\
\left({ }^{\circ} \mathrm{C}\right)\end{array}$} & \multicolumn{4}{|c|}{ EWL (in \%) } \\
\hline & & & after 7 days & after 14 days & after 21 days & after 28 days \\
\hline 1 & 2 & 3 & 4 & 5 & 6 & 7 \\
\hline CGS & & & 0.95 & $1.77 \mathrm{ab}$ & $2.68 \mathrm{ab}$ & $3.42 \mathrm{~b}$ \\
\hline GP & & & 0.87 & $1.60 \mathrm{~b}$ & $2.48 \mathrm{~b}$ & $3.40 \mathrm{~b}$ \\
\hline $\mathrm{CH}$ & & & 0.95 & $1.84 \mathrm{a}$ & $2.96 \mathrm{a}$ & $3.95 \mathrm{a}$ \\
\hline \multirow[t]{9}{*}{ WL } & & & 1.00 & $1.74 \mathrm{ab}$ & $2.73 \mathrm{ab}$ & $3.58 \mathrm{ab}$ \\
\hline & 0 & & - & - & - & - \\
\hline & 14 & & $0.87 \mathrm{~b}$ & 1.79 & - & - \\
\hline & 28 & & $1.02 \mathrm{a}$ & 1.69 & 2.71 & 3.59 \\
\hline & & fresh & - & - & - & - \\
\hline & & 5 & $0.59 \mathrm{~b}$ & $0.99 \mathrm{~b}$ & $1.43 \mathrm{~b}$ & $1.93 \mathrm{~b}$ \\
\hline & & 20 & $1.30 \mathrm{a}$ & $2.49 \mathrm{a}$ & $4.00 \mathrm{a}$ & $5.24 \mathrm{a}$ \\
\hline & 0 & fresh & - & - & - & - \\
\hline & 14 & 5 & 0.50 ef & 0.97 & - & - \\
\hline \multirow{5}{*}{ CGS } & 14 & 20 & $1.26 \mathrm{ab}$ & 2.64 & - & - \\
\hline & 28 & 5 & $0.80 \mathrm{de}$ & 0.86 & 1.24 & 1.60 \\
\hline & 20 & 20 & $1.25 \mathrm{ab}$ & 2.60 & 4.12 & 5.23 \\
\hline & 0 & fresh & - & - & - & - \\
\hline & 14 & 5 & 0.52 ef & 1.06 & - & - \\
\hline \multirow[t]{5}{*}{ GP } & 14 & 20 & $1.24 \mathrm{abc}$ & 2.17 & - & - \\
\hline & 28 & 5 & 0.49 ef & 0.95 & 1.45 & 1.93 \\
\hline & 28 & 20 & $1.24 \mathrm{abc}$ & 2.21 & 3.50 & 4.87 \\
\hline & 0 & fresh & - & - & - & - \\
\hline & 14 & 5 & $0.25 \mathrm{f}$ & 0.94 & - & - \\
\hline \multirow[t]{5}{*}{$\mathrm{CH}$} & 17 & 20 & $1.42 \mathrm{a}$ & 2.93 & - & - \\
\hline & 28 & 5 & $0.90 \mathrm{~cd}$ & 1.01 & 1.46 & 2.10 \\
\hline & & 20 & $1.21 \mathrm{abc}$ & 2.49 & 4.47 & 5.81 \\
\hline & 0 & fresh & - & - & - & - \\
\hline & 14 & 5 & $0.27 \mathrm{f}$ & 1.02 & - & - \\
\hline \multirow[t]{3}{*}{ WL } & 14 & 20 & $1.46 \mathrm{a}$ & 2.57 & - & - \\
\hline & 28 & 5 & $0.97 \mathrm{bcd}$ & 1.11 & 1.57 & 2.09 \\
\hline & 20 & 20 & $1.29 \mathrm{ab}$ & 2.28 & 3.90 & 5.06 \\
\hline
\end{tabular}




\begin{tabular}{|c|c|c|c|c|c|c|}
\hline 1 & 2 & 3 & 4 & 5 & 6 & 7 \\
\hline \multicolumn{7}{|l|}{ P-value } \\
\hline $\mathrm{G}$ & & & 0.5521 & $\leq 0.05$ & $\leq 0.05$ & $\leq 0.05$ \\
\hline STI & & & $\leq 0.05$ & 0.1506 & - & - \\
\hline STE & & & $\leq 0.0001$ & $\leq 0.0001$ & $\leq 0.0001$ & $\leq 0.0001$ \\
\hline $\mathrm{G} \times \mathrm{STI}$ & & & 0.3867 & 0.8792 & 0.0648 & 0.1825 \\
\hline $\mathrm{G} \times \mathrm{STE}$ & & & 0.8369 & $\leq 0.05$ & - & - \\
\hline $\mathrm{STI} \times \mathrm{STE}$ & & & $\leq 0.0001$ & 0.2374 & - & - \\
\hline $\mathrm{G} \times \mathrm{STI} \times \mathrm{STE}$ & & & $\leq 0.05$ & 0.2720 & - & - \\
\hline SEM & & & 0.037 & 0.055 & 0.122 & 0.155 \\
\hline
\end{tabular}

G - genotype; STI - storage time; STE - storage temperature; EWL - egg weight loss; CGS - Czech Golden Spotted hen; GP - Greenleg Partridge; CH - commercial hybrid; WL - White Leghorn; SEM - standard error of mean; Values with significance of P $\leq 0.05$ were considered as significant.

\section{Microbial contamination}

Regarding the eggshell contamination (Table 1), the effect of genotype was found to be statistically significant in all three observed groups of microorganisms. The significantly lowest contamination of eggshell by EC was in eggs from the WL hens (4.42 log CFU/eggshell), while from ENT was in eggs from the CGS hens (1.22 $\log$ CFU/eggshell) and from the WL hens (1.40 log CFU/ eggshell). The lowest incidence of TNM was also detected in eggs from the WL hens (5.03 log CFU/eggshell). These data obviously show that in terms of genotype, WL hens achieved the most favourable results compared to other selected genotypes. The effect of storage time on eggshell contamination was determined as significant only in contamination by ENT bacteria, where the lowest level of contamination was found in eggs stored for 28 days (1.16 log CFU/eggshell). However, the decreasing trend of the eggshell contamination with the increasing storage time was detected in all evaluated groups of microorganisms (EC, ENT and TNM). This trend was regular in contamination by ENT and TNM, while irregular in contamination by EC. When comparing the effect of storage temperature on microbial contamination of the eggshell, the contamination by ENT and by TNM were calculated as statistically significant. The lowest levels were found in eggs stored at $20^{\circ} \mathrm{C}$, specifically $1.12 \mathrm{log}$ CFU/eggshell for ENT and $5.00 \log$ CFU/eggshell for TNM. The level of microbial contamination regularly decreased with the increasing storage temperature in all monitored groups of microorganisms. Furthermore, there were several interactions calculated as significant. Specifically, the interaction between genotype and storage temperature in contamination by ENT and TNM and the interaction between storage time and temperature in contamination by TNM. The statistically lowest eggshell contamination by ENT was determined in eggs from the CGS hens stored at $20^{\circ} \mathrm{C}(0.20 \log \mathrm{CFU} /$ eggshell $)$ and the highest in fresh eggs from the $\mathrm{CH}$ hens (3.36 log CFU/ eggshell). The lowest incidence of TNM on eggshell was determined in eggs from the GP hens stored at $20^{\circ} \mathrm{C}(4.16$ $\log$ CFU/eggshell) and the highest in fresh eggs from $\mathrm{CH}$ hens (6.56 log CFU/eggshell). In terms of the interaction between storage time and temperature, which was found significant only in contamination by TNM, the lowest value was found in eggs stored for 28 days at $20^{\circ} \mathrm{C}$ (4.42 log CFU/eggshell) and the highest in fresh eggs (6.06 log CFU/eggshell).

The microbial contamination of eggshell membranes (Table 2) was significantly affected by genotype (TNM) and storage time (EC and TNM). Statistically the lowest contamination of eggshell membranes by TNM was found in eggs from the WL $(0.12 \log$ CFU/eggshell membranes) and $\mathrm{CH}$ hens (0.15 log CFU/eggshell membranes). When considering the effect of storage time, the significantly lowest contamination of eggshell membranes by EC was found in fresh eggs (not detected) and in eggs stored for 28 days (0.07 log CFU/eggshell membranes). Storage time significantly affected the contamination by TNM, where the lowest incidence of microorganisms in eggshell membranes was found in eggs stored for 28 days (0.23 log CFU/eggshell membranes) and in fresh eggs (0.28 log CFU/eggshell membranes). The statistically significant interaction between genotype and storage time was determined in contamination by TNM, where the lowest value was detected in eggshell membranes from the WL eggs stored for 28 days (not detected) and from the $\mathrm{CH}$ fresh eggs (not detected). Vice versa, the highest value was detected in eggshell membranes from the CGS and GP hens stored for 14 days (1.45 and $1.41 \log$ CFU/eggshell membranes).

The genotype and interaction between genotype and storage temperature were determined as significant in microbial contamination of albumen by EC (Table 3 ). Regarding the effect of genotype, the GP (not detected) and WL (not detected) hens had eggs with statistically the lowest occurrence of EC bacteria in albumen. The interaction between genotype and storage temperature showed that the lowest contamination of EC in albumen was in eggs from all storage temperature groups from the GP and WL hens (not detected). Moreover, fresh eggs from the CGS and $\mathrm{CH}$ hens and eggs from the $\mathrm{CH}$ stored at $5^{\circ} \mathrm{C}$ had also the same level of contamination by EC 
(not detected). On the other hand, the highest contamination of albumen was in eggs from the CGS hens stored at a temperature of $5^{\circ} \mathrm{C}(1.18 \log \mathrm{CFU} /$ albumen $)$.

\section{Egg weight and eggshell quality parameters}

The significant effects of genotype, storage time and temperature were determined in EW and in some eggshell quality parameters (Table 4). Moreover, significant interactions were calculated as well. The EW and all observed eggshell parameters were significantly affected by the genotype. The heaviest eggs were from the commercial hybrid hens $(57.97 \mathrm{~g})$. The highest value of the SP $(9.72 \%)$ and the ST $(0.314 \mathrm{~mm})$ was determined in eggs from the WL hens. The strongest eggshells were from the CGS hens $\left(42.70 \mathrm{~N} / \mathrm{cm}^{2}\right)$. The highest value of the SI was from the WL hens $\left(7.94 \mathrm{~g} / 100 \mathrm{~cm}^{2}\right)$. The last eggshell parameter, which was significantly influenced by genotype was the ESS and the highest was in eggs from the $\mathrm{CH}$ hens $\left(70.08 \mathrm{~cm}^{2}\right)$. The effect of storage time was determined as significant in the EW, SP, ST, SI and SS. The heaviest eggs were fresh eggs (57.96 g). The highest value of the SP was found in eggs stored for 28 days $(9.51 \%)$. The ST value was determined highest in eggs stored for 28 days $(0.311 \mathrm{~mm})$ and in fresh eggs $(0.309 \mathrm{~mm})$. Storage time had also a significant effect on the SI, which was found as highest in eggs stored for 28 days $\left(7.76 \mathrm{~g} / 100 \mathrm{~cm}^{2}\right)$. The highest value of the SS was found in fresh eggs $\left(70.08 \mathrm{~cm}^{2}\right)$. The effect of storage temperature was statistically significant in exactly the same parameters as the effect of storage time. Significantly heaviest eggs were fresh eggs (57.96 g). The SP was found to be the highest in eggs stored at $20^{\circ} \mathrm{C}$. The highest value of the ST was in eggs stored at $5^{\circ} \mathrm{C}$ $(0.310 \mathrm{~mm})$ and fresh eggs $(0.309 \mathrm{~mm})$. The SI was determined to be the highest in eggs stored at $5^{\circ} \mathrm{C}(7.78$ $\left.\mathrm{g} / 100 \mathrm{~cm}^{2}\right)$ and at $20^{\circ} \mathrm{C}\left(7.74 \mathrm{~g} / 100 \mathrm{~cm}^{2}\right)$. The highest value of the SS was determined in fresh eggs $\left(70.08 \mathrm{~cm}^{2}\right)$.

The interactions between genotype and storage time and between genotype and storage temperature were calculated as being significant in the same parameters, specifically in the EW, SP, ST, SI and SS. Regarding the interaction between genotype and storage time, the heaviest eggs were fresh eggs from $\mathrm{CH}$ hens $(60.19 \mathrm{~g})$, while the lightest eggs were eggs from the GP hens stored for 28 days $(54.89 \mathrm{~g})$. The highest value of the interaction between genotype and storage time in SP was calculated in eggs from the WL hens stored for 14 days $(9.77 \%)$ and the lowest in fresh eggs from the GP hens $(8.79 \%)$ and in eggs from the GP and $\mathrm{CH}$ hens stored for 14 days ( 8.81 and $8.88 \%$, respectively). The same interaction significantly affected ST, where the thickest eggshell was in eggs from $\mathrm{CH}$ hens stored for 28 days $(0.325$ $\mathrm{mm})$ and the thinnest eggs from $\mathrm{CH}$ stored for 14 days $(0.289 \mathrm{~mm})$ and eggs from the GP hens stored for 28 and 14 days, respectively ( 0.290 and $0.292 \mathrm{~mm}$, respectively). The interaction between genotype and storage time was determined also in SI, where the highest value was found in eggs from $\mathrm{CH}$ hens stored for 28 days $(7.95 \mathrm{~g} / 100$ $\mathrm{cm}^{2}$ ) and the lowest in eggs from the GP hens stored for 14 days $\left(7.21 \mathrm{~g} / 100 \mathrm{~cm}^{2}\right)$. The last eggshell parameter that was significantly affected by this interaction was SS. The significantly highest value of SS was in fresh eggs from the $\mathrm{CH}$ hens $\left(71.87 \mathrm{~cm}^{2}\right)$, while the lowest was in eggs from the GP hens stored for 28 days $\left(67.58 \mathrm{~cm}^{2}\right)$. The significant effect of the interaction between genotype and storage temperature was found in EW. The heaviest eggs were fresh eggs from the $\mathrm{CH}(60.19 \mathrm{~g})$, while the lightest eggs were eggs from the GP stored at $20^{\circ} \mathrm{C}(54.59 \mathrm{~g})$. In terms of the interaction between genotype and storage temperature, SP was calculated as significant, where the highest value was determined in eggs from the WL hens stored at $5^{\circ} \mathrm{C}(9.84 \%)$ and the lowest value in fresh eggs from the GP hens $(8.79 \%)$ and eggs stored at of $5^{\circ} \mathrm{C}(8.83 \%)$. As mentioned before, the statistically significant effect of this interaction was calculated also in $\mathrm{ST}$, where the thickest eggshells were in eggs from the WL hens stored at $5^{\circ} \mathrm{C}(0.329 \mathrm{~mm})$ and the thinnest were in eggs from the GP hens stored at $20^{\circ} \mathrm{C}(0.286 \mathrm{~mm})$. The significantly highest value of SI was found in eggs from the WL hens stored at $5^{\circ} \mathrm{C}\left(8.00 \mathrm{~g} / 100 \mathrm{~cm}^{2}\right)$ and the lowest in eggs from all storage temperature groups of eggs (fresh, $5^{\circ} \mathrm{C}$ and $20^{\circ} \mathrm{C}$ ) from the GP hens $(7.22,7.24$ and $7.32 \mathrm{~g} / 100 \mathrm{~cm}^{2}$, respectively). The last eggshell parameter influenced by the interaction between genotype and storage temperature was SS. The highest value was calculated in fresh eggs from the $\mathrm{CH}$ hens $\left(71.87 \mathrm{~cm}^{2}\right)$ and the lowest in eggs from the GP hens stored at $20^{\circ} \mathrm{C}$ $\left(67.33 \mathrm{~cm}^{2}\right)$. Moreover, the interaction between storage time and storage temperature was determined in EW, ST and SS. The heaviest eggs were fresh eggs (57.96 g), while the lightest were eggs stored for 28 days at $20^{\circ} \mathrm{C}$ $(54.64 \mathrm{~g})$. The ST was significantly the highest in eggs stored for 28 days at $5^{\circ} \mathrm{C}(0.312 \mathrm{~mm})$ and $20^{\circ} \mathrm{C}(0.309$ $\mathrm{mm})$, fresh eggs $(0.309 \mathrm{~mm})$ and eggs stored for 14 days at $5^{\circ} \mathrm{C}(0.308 \mathrm{~mm})$. Vice versa, the statistically thinnest eggshell was found in eggs stored for 14 days at $20^{\circ} \mathrm{C}$ $(0.286 \mathrm{~mm})$. The last parameter, where the significant interaction between storage time and storage temperature was calculated, was SS. The highest value of SS was in fresh eggs $\left(70.08 \mathrm{~cm}^{2}\right)$ and the lowest was in eggs stored for 28 days at $20^{\circ} \mathrm{C}\left(67.37 \mathrm{~cm}^{2}\right)$.

Regarding the EWL, significant effect of genotype, storage time, storage temperature and their mutual interactions were calculated (Table 5). Statistically significant effect of genotype on EWL was found in eggs after 14, 21 and 28 days of storage. The lowest EWL at the end of the storage period was in eggs from GP and CGS hens (3.40 and $3.42 \%$, respectively) and the highest in eggs from $\mathrm{CH}$ hens $(3.95 \%)$. As expected, the effect of storage time and storage temperature influenced EWL. Specifically, EWL regularly increased with storage time and simultaneously, higher EWL was determined in eggs stored at $20^{\circ} \mathrm{C}$ compared to $5^{\circ} \mathrm{C}$. Significant interaction between storage time and temperature was found in EWL in eggs after 7 days of storage, where the highest EWL was found in eggs stored for 14 and 28 days at $20^{\circ} \mathrm{C}(1.35$ 
and $1.32 \%$, respectively) and the lowest in eggs stored for 14 days at $5^{\circ} \mathrm{C}(0.39 \%)$. Significant interaction between genotype and storage temperature was determined in EWL in eggs after 14 days of storage, where the highest EWL was found in eggs from $\mathrm{CH}$ hens stored at $20^{\circ} \mathrm{C}$ $(2.71 \%)$ and the lowest in eggs from CGS, CH, GP and WL hens all stored at $5^{\circ} \mathrm{C}(0.92,0.98,1.01$ and $1.06 \%$, respectively). The only significant three-way interaction among genotype, storage time and storage temperature for EWL was calculated in eggs after 7 days of storage. The only three-way interaction (among genotype, storage time and temperature) in EWL, which was calculated as statistically significant, was found in eggs after 7 days of storage, where the highest EWL was in eggs from WL and $\mathrm{CH}$ hens stored for 14 days at $20^{\circ} \mathrm{C}(1.46$ and $1.42 \%$, respectively) and the lowest was in eggs from $\mathrm{CH}$ and WL hens stored for 14 days at $5^{\circ} \mathrm{C}(0.25$ and $0.27 \%$, respectively).

\section{Discussion}

The effect of storage time or storage temperature on microbial contamination, respectively on microbial penetration into the egg content was previously observed by a number of studies, for example by Stepien-Pysniak (2010) and Vlčková et al. (2018). However, the effect of genotype was not studied in as much depth as other factors. Authors such as De Reu et al. (2008) and Englmaierová et al. (2014) usually focus on the effect of different housing systems, but not on the effect of genotype. Nevertheless, Jones et al. (2004) carried out a study, where the effect of genotype on microbial contamination of eggs was not only observed, but also confirmed. Moreover, these authors concluded that genetic selection has negatively affected the resistance of eggs to microbial contamination and penetration through the eggshell during the storage time. This statement can be supported by the findings of our study, where the differences in the microbial contamination and penetration among the genotypes were found. Specifically, the results showed the most favourable values for eggs from WL hens, where the statistically lowest eggshell contamination was found in all monitored groups of microorganisms and also the lowest in contamination of eggshell membranes and albumen, when values were significant. Vice versa, the worst results were found in eggs from the CGS hens, which may have been caused by the fact that these hens belong to genetic resources of the Czech Republic and the selection is based on their exterior signs. These findings can be supported by the previously mentioned statement from Jones et al. (2004), which claims that genotype has an effect on microbial contamination of eggs. Jones et al. (2015) add that the higher contamination and subsequent penetration might be caused also by the behavioural patterns, for example by behaviour connected to oviposition. They found out that the higher contamination occurs in eggs laid on the floor compared to nest boxes. However, that is not the case of our study, because there were only eggs used from nest boxes. Furthermore, Kusuda et al. (2011) observed the diversity in the cuticle structure among different avian species including the Japanese quail (Coturnix japonica), Red Junglefowl (Gallus gallus), Greater Flamingo (Phoenicopterus ruber roseus), White Pelican (Pelecanus onocrotalus) and Humboldt Penguin (Spheniscus humboldti). The eggshell cuticle represents the first defensive barrier of the egg to avoid penetration of undesirable pathogens into the eggshell content. The thickness of the cuticle and its coverage on the eggshell surface are traits that are heritable (Kulshreshtha et al., 2018). The results of Kusuda et al. (2011) indicated that the differences in cuticle structure, coverage and functionality occurred in different avian species. Therefore, the same pattern could possibly work for different genotypes of hens. When considering the effect of genotype, the microbial contamination of eggshell membranes and albumen did not show corresponding values to the contamination of the eggshell surface. Specifically, there was almost no detection of penetration of microorganisms into the egg content in the eggs from $\mathrm{CH}$ hens despite a high initial contamination of egg surface. Conversely, microorganisms (especially EC and TNM) occurred in egg content of eggs from native breeds. These results may suggest a better antimicrobial defence of eggshell membranes, respectively of albumen. The study from Lewko and Gornowicz (2009) supports this statement, because they found differences in the content and activity of lysozyme among different hen genotypes. According to You et al. (2010) lysozyme is the most represented (the content of lysozyme in albumen is $3.5 \%$ ) and the most effective tool of albumen against the contamination of egg content by pathogens. Lysozyme is included not only in albumen, but also in eggshell membranes and in eggshell itself (Hincke et al., 2000). Furthermore, lysozyme is effective especially against gram-positive bacteria (Hincke et al., 2000), so the low levels of ENT bacteria (gram-positive) observed in this study compared to other groups might be caused by the activity of lysozyme. Vlčková et al. (2019) add that ovotransferrin and ovalbumin are other proteins that play an essential role in terms of the antimicrobial defence of albumen and that ovotransferrin concentration is influenced by genetics. This also supports the idea of different level of antimicrobial defence of eggs from different hen genotypes. Furthermore, the findings of Vlčková et al. (2018) also concluded that the level of contamination of eggshell and penetration into egg content by ENT was lower in comparison with EC and TNM. Despite the highest initial contamination of eggshell surface of eggs from $\mathrm{CH}$ hens, the occurrence on the eggshell membranes and in albumen was statistically or numerically the lowest compared to native breeds. Considering the discussed literature, the results of this study may indicate that eggs from $\mathrm{CH}$ hens are less prone to microbial penetration through the eggshell or that these hens produce eggs with better antimicrobial defence. The results of various studies (Park et 
al., 2003; Aygun and Sert, 2013 a and Vlčková et al., 2018) showed the decrease of microbial contamination of eggshell surface with the increasing storage time regardless of the origin of the microorganisms. This is in accordance with the findings of our study, where the lowest levels of microbial contamination were found in eggs stored for 28 days, when significant. The statistically highest incidence of microorganisms on the eggshell membranes was detected in the eggs stored for 14 days, with the exception of ENT, where the effect of storage time was non-significant and the values were very low. Very similar results were achieved by Vlčková et al. (2018) including the overall low contamination by ENT, but their results were not statistically significant. Nevertheless, the similar trends of the results are obvious. The same trend relates to contamination of albumen, but our results were statistically non-significant and the values were lower compared to contamination of eggshell membranes. The reason why the ENT values are usually low was already discussed above. Regarding the effect of storage temperature on microbial contamination of eggs, the higher temperatures are less favourable than the lower temperatures from the perspective of egg quality and safety (Theron et al., 2003). The ideal temperature for the growth of EC bacteria ranges from 20 to $37^{\circ} \mathrm{C}$. The higher the temperature (when the range from 20 to $37^{\circ} \mathrm{C}$ is taken into account), the faster the growth (Farewell and Neidhardt, 1998). The range of temperatures where ENT bacteria are capable of growing, varies, the optimal temperature for ENT growth is $42.7^{\circ} \mathrm{C}$, the minimum is $6.5^{\circ} \mathrm{C}$ and the maximum is $47.8^{\circ} \mathrm{C}$ (Fisher and Phillips, 2009). The significant effect of storage temperature was found only in the contamination of egg surface. The highest contamination levels were found in fresh eggs compared to eggs stored at two different temperatures (5 and $20^{\circ} \mathrm{C}$ ), which corresponds with the storage time and decreasing trend of microorganisms with extended storage time. The only significant difference between the two observed temperatures was found in eggshell contamination by ENT, where lower values were detected in eggs stored at $20^{\circ} \mathrm{C}$. Other differences between these storage temperatures were determined as non-significant. The similar growth of observed microorganism groups in both storage temperatures could be caused by the fact that the temperatures $\left(5\right.$ and $\left.20^{\circ} \mathrm{C}\right)$ are far from the ideal temperature for growth so therefore there was not any significant difference detected between these storage temperatures. The calculated interactions between genotype and storage time, respectively storage temperature, reflect the individual relationship of the specific genotype to the storage conditions (time or temperature). These interactions should be further studied to draw substantiated conclusions. However, an interesting interaction between storage time and storage temperature was detected in eggshell contamination by TNM. This interaction revealed that the best results of the combination of storage time and storage temperature regarding the eggshell contamination, was storage for 28 days at $20^{\circ} \mathrm{C}$, which is controversial because of a generally known fact that the egg quality deteriorates with prolonged storage time.

The importance of eggshell quality is multilateral, it represents the protection of egg content against microbial contamination in terms of food safety (Zaheer, 2015), respectively in terms of hatchability in hatching eggs (Yamak et al., 2016). Another substantial effect of eggshell is connected to the economics, because the eggs with damaged eggshell represent from 6 to $8 \%$ of overall egg production (Bain et al., 2006). Usually, the eggshell quality is defined by its weight, proportion and thickness (Messens et al., 2005). Bain et al. (2006) consider also the strength as the one of the most essential eggshell quality parameters. Eggs with thinner eggshells are more likely to be penetrated by pathogenic microorganisms (Messens et al., 2005). Moreover, the cuticle contributes to the eggshell thickness according to Kusuda et al. (2011). The significance of cuticle regarding the microbial contamination was discussed above in detail. The effect of genotype on EW and various eggshell quality parameters was previously confirmed by various authors such as Zita et al. (2009), Khatun et al. (2016) and Kraus et al. (2020). Also, a large number of studies (e.g. Akter et al., 2014; Krawczyk and Sokołowicz, 2015; Vlčková et al., 2019) that were focused on the effect of storage conditions (storage time, storage temperature or both), was carried out in the past. The inconsistencies in some results among the studies could be caused by the different use of genotypes, storage times, and storage temperatures and also by other factors, such as housing systems, feed mixtures etc. However, our results predominantly correspond to the general and previously confirmed facts and trends. The interactions of EW and eggshell parameters between genotype and storage time or storage temperature are to a large extent influenced by the individuality of each specific genotype (same as for the interactions of microbial by various microorganisms). Nevertheless, the interaction between storage time and temperature, which could reveal some trends, was calculated in EW, ST and $\mathrm{SS}$. The results of this interaction in EW and SS were exactly the same, the highest values were determined in fresh eggs and the lowest in eggs stored for 28 days at $20^{\circ} \mathrm{C}$. This is probably caused by the fact that $\mathrm{EW}$ values are used for the calculation of SS. Lee et al. (2016) confirmed the same trends in EW and also found the interaction between storage time and temperature as significant. Specifically, these authors determined the decrease with an extended storage time and the decrease with an increased storage temperature. The interaction between storage time and temperature in ST showed that the lowest value of ST was found in eggs stored for 14 days at $20^{\circ} \mathrm{C}$. Samli et al. (2005) also found the significant effect of this interaction on ST, but the trend is not as obvious as in the EW or SS.

The effect of storage conditions on EWL was previously studied by a large number of authors, such as Sert et al. (2011), Aygun and Sert (2013 b) and Brodacki et al. (2019). Specifically the study from Aygun and Sert 
(2013 b) focused on the comparison of two storage treatments (control and vacuum) during different storage times and temperatures. The results of their study, same as the results of this study, showed that prolonged storage time and higher storage temperature (storage temperatures 5 and $22^{\circ} \mathrm{C}$ were studied) negatively influences EWL. Brodacki et al. (2019) concluded that the use of lower storage temperatures is more suitable for egg storage because it prolonged the shelf life of eggs, which is in accordance with the results of our study.

\section{Conclusion}

The most essential finding of this study is that there were significant differences in microbial contamination of egg surface among observed hen genotypes. The penetration of selected microorganisms was also significant in contamination of eggshell membranes by TNM and in contamination of albumen by EC. Regarding these results, breeders should focus not only on the performance parameters of hens, but also on the ability of eggs to resist the microbial contamination and penetration during storage because of the safety of eggs.

Specifically, regarding the effect of genotype on microbial contamination and penetration, it can be concluded that WL hens achieved the best results among the selected genotypes based on the obtained results. Regarding the effect of storage time, from the results it is obvious that in terms of microbial contamination it is suitable to store eggs for 28 days, but on the other hand it is not compatible with quality and especially with the freshness of egg content. These results confirm the generally known fact that the level of eggshell contamination decreases with time. In terms of storage temperature, there were not enough significant results calculated to make any statement. Furthermore, there were several two-way interactions calculated as statistically significant $(\mathrm{G} \times \mathrm{STI}, \mathrm{G} \times \mathrm{STE}$ and STI $\times$ STE $)$, nevertheless there were not any patterns found so it is impossible to draw any conclusions. Only the interaction between storage time and storage time and temperature showed interesting results, where the lowest contamination of eggshell was determined in eggs stored for 28 days at $20^{\circ} \mathrm{C}$. Speaking of the egg weight and egg quality parameters, it is impossible to unequivocally choose the best genotype. However, the results of eggs from WL hens seem to be the best overall. The most notable changes caused by storage time and temperature were found in EW (and logically in SP, which is in direct relationship with EW). The higher the storage time (storage temperature, resp.) was, the lower the EW was. The results of SP were exactly the opposite. Based on the findings of this study, it can be recommended to store eggs at lower storage temperatures for a short time to avoid high EWL. Regarding the genotype, the lowest EWL during the storage time was achieved in eggs from GP hens, followed by eggs from CGS hens.

\section{Acknowledgements}

The funding was provided by an "S" grant of Ministry of Education, Youth and Sports of the Czech Republic and grant project no. SV21-6-21320. Furthermore, we are grateful to Mr. Richard Hardy for the language correction of the manuscript.

\section{References}

Ahmed A.M.H., Rodríguez-Navarro A.B., Vidal M.L., Gautron J., García-Ruiz J.M., Nys Y. (2005). Changes in eggshell mechanical properties, crystallographic texture and in matrix proteins induced by moult in hens. Brit. Poultry Sci., 46: 268-279.

Akter Y., Kasim A., Omar H., Sazili A.Q. (2014). Effect of storage time and temperature on the quality characteristics of chicken eggs. J. Food Agric. Environ., 12: 87-92.

Anderle V., Lichovníková M., Przywarová A., Dračková E. (2014). Egg quality of gene reserve Czech Golden Spotted hens. Acta Fytotech. Zootech., 17: 84-86.

Aygun A., Sert D. (2013 a). Effects of prestorage application of propolis and storage time on eggshell microbial activity, hatchability, and chick performance in Japanese quail (Coturnix coturnix japonica) eggs. Poultry Sci., 92: 3330-3337.

Aygun A., Sert D. (2013 b). Effects of vacuum packing on eggshell microbial activity and egg quality in table eggs under different storage temperatures. J. Sci. Food Agric., 93: 1626-1632.

Bain M.M., Dunn I.C., Wilson P.W., Joseph N., De Ketelaere B., De Baerdemaeker J., Waddington D. (2006). Probability of an egg cracking during packing can be predicted using a simple non-destructive acoustic test. Brit. Poultry Sci., 47: 462-469.

Batkowska J., Brodacki A. (2017). Selected quality traits of eggs and the productivity of newly created laying hen hybrids dedicated to an extensive rearing system. Arch. Anim., 60: 87-93.

Brodacki A., Batkowska J., Drabik K., Chabroszewska P., Łuczkiewicz P. (2019). Selected quality traits of table eggs depending on storage time and temperature. Brit. Food J., 121: 2016-2026.

D’Alba L., Shawkey M.D. (2015). Mechanisms of antimicrobial defense in avian eggs. J. Ornithol., 156: 399-408.

De Reu K., Messens W., Heyndrickx M., Rodenburg T.B., Uyttendaele M., Herman L. (2008). Bacterial contamination of table eggs and the influence of housing systems. World. Poultry Sci. J., 64: 5-19.

Dikmen B.Y., İpek A., Şahan Ü., Petek M., Sözcü A. (2016). Egg production and welfare of laying hens kept in different housing systems (conventional, enriched cage, and free range). Poultry Sci., 95: 1564-1572.

Elmi M. (2004). Food safety: current situation, unaddressed issues and the emerging priorities. East. Mediterr. Health J., 10: 794-800.

Englmaierová E., Tůmová E., Charvátová V., Skřivan M. (2014). Effects of laying hens housing system on laying performance, egg quality characteristics, and egg microbial contamination. Czech J. Anim. Sci., 59: 345-352.

FAO (2003). Assuring food safety and quality: guidelines for strengthening national food control systems. Food and Agriculture Organization of the United Nations and World Health Organization Rome (Italy).

FAO (2007). The state of world's animal genetic resources for food and agriculture. Commission on Genetic Resources for Food and Agriculture Food; Food and Agriculture Organization of the United Nations Rome (Italy).

Farewell A., Neidhardt F.C. (1998). Effect of temperature on in vivo protein synthetic capacity in Escherichia coli. J. Bacteriol., 180: 4704-4710.

Fisher K., Phillips C. (2009). The ecology, epidemiology and virulence of Enterococcus. Microbiology, 155: 1749-1757.

Hanusová E., Hrnčár C., Hanus A., Oravcova M. (2015). Effect of 
breed on some parameters of egg quality in laying hens. Acta Fytotech. Zootech., 18: 20-24.

Hanusová E., Hrnčár C., Hanus A., Ondruška L. (2017). Characterization of native Slovak chicken and goose: a review. Slovak J. Anim. Sci., 50: 144-148.

Hernandez J.M., Beardswort P.M., Weber G. (2005). Egg quality meeting consumer expectations. Int. Poultry Prod., 13: 20-23.

Hincke M.T., Gautron J., Panheleux M., Garcia-Ruiz J., McKee M.D., Nys Y. (2000). Identification and localization of lysozyme as a component of eggshell membranes and eggshell matrix. Matrix Biol., 19: 443-453.

Hy-Line (2021). Hy-Line Brown: The World's Most Balanced Egg Layer. Management Guide. https:/www.hyline.com/varieties/ brown

Iannotti L.L., Lutter C.K., Bunn D.A., Stewart C.P. (2014). Eggs: the uncracked potential for improving maternal and young child nutrition among the world's poor. Nutr. Rev., 72: 355-368.

Jones D.R., Anderson K.E., Davis G.S. (2001). The effects of genetic selection on production parameters of single comb White Leghorn hens. Poultry Sci., 80: 1139-1143.

Jones D.R., Curtis P.A., Anderson K.E., Jones F.T. (2004). Microbial contamination in inoculated shell eggs: II. Effects of layer strain and egg storage. Poultry Sci., 83: 95-100.

Jones D.R., Cox N.A., Guard J., Fedorka-Cray P.J., Buhr R.J., Gast R.K., Abdo Z., Rigsby L.L., Plumblee J.R., Karcher D.M., Robison C.I., Blatchford R.A., Makagon M.M. (2015). Microbiological impact of three commercial laying hen housing systems. Poultry Sci., 94: 544-551.

Khatun H., Rashid M.A., Faruque S., Islam M.N., Ali M.Y. (2016) Study on egg quality characteristics of three commercial laye strains under different storage conditions. Int. J. Anim. Res., 1: $63-70$.

Kraus A., Zita L., Krunt O. (2019). The effect of different housing system on quality parameters of eggs in relationship to the age in brown egg-laying hens. Bulg. J. Agric. Sci., 25: 1246-1253.

Kraus A., Zita L., Krunt O., Pokorná K. (2020). How genotype influences the egg quality in the second half of laying cycle? J. Cent. Eur. Agric., 21: 215-221.

Kraus A., Zita L., Krunt O., Härtlová H., Chmelíková E. (2021). Determination of selected biochemical parameters in blood serum and egg quality of Czech and Slovak native hens depending on the housing system and hen age. Poultry Sci., 100: 1142-1153.

Krawczyk J. (2009). Quality of eggs from Polish native Greenleg Partridge chicken-hens maintained in organic vs. backyard production systems. Anim. Sci. Pap. Rep., 27: 227-235.

Krawczyk J., Sokołowicz Z. (2015). Effect of chicken breed and storage conditions of eggs on their quality. Acta Sci. Pol. Zootech. 14: $109-118$.

Krawczyk J., Sokołowicz Z., Szymczyk B. (2011). Effect of housing system on cholesterol, vitamin and fatty acid content of yolk and physical characteristics of eggs from Polish native hens. Arch. Geflugelkd., 75: 151-157.

Krunt O., Zita L., Kraus A., Okrouhlá M., Chodová D., Stupka R. (2021). Guinea fowl (Numida meleagris) eggs and free range housing: A convenient alternative to laying hens' eggs in terms of food safety? Poultry Sci., 100: 101006.

Kulshreshtha G., Rodriguez-Navarro A., Sanchez-Rodriguez E., Diep T., Hincke M.T. (2018). Cuticle and pore plug properties in the table egg. Poultry Sci., 97: 1382-1390.

Kusuda S., Iwasawa A., Doi O., Ohya Y., Yoshizaki N. (2011). Diversity of the cuticle layer of avian eggshells. J. Poultry Sci., 48: $119-124$
Lee M.H., Cho E.J., Choi E.S., Sohn S.H. (2016). The effect of storage period and temperature on egg quality in commercial eggs. Korean J. Poultry Sci., 43: 31-38.

Lewko L., Gornowicz E. (2009). Egg albumen quality as affected by bird origin. J. Cent. Eur. Agric., 10: 455-463.

Messens W., Grijspeerdt K., Herman L. (2005). Eggshell penetration by Salmonella: a review. World. Poultry Sci. J., 61: 71-86.

Moyle T., Drake K., Gole V., Chousalkar K., Hazel S. (2016). Bacterial contamination of eggs and behaviour of poultry flocks in the free range environment. Comp. Immunol. Microbiol. Infect. Dis., 49: 88-94.

Park Y.S., Yoo I.J., Jeon K.H., KimChang E.J., Oh H.I. (2003). Effects of various eggshell treatments on the egg quality during storage. Asian-Australas. J. Anim. Sci., 16: 1224-1229.

Pohle K., Cheng H.W. (2009). Comparative effects of furnished and battery cages on egg production and physiological parameters in White Leghorn hens. Poultry Sci., 88: 2042-2051.

Roberts J.R. (2004). Factors affecting egg internal quality and egg shell quality in laying hens. J. Poultry Sci., 41: 161-177.

Rodríguez-Navarro A.B., Domínguez-Gasca N., Muñoz A., OrtegaHuertas M. (2013). Change in the chicken eggshell cuticle with hen age and freshness. Poultry Sci., 92: 3026-3035.

Samli H.E., Agma A., Senkoylu N. (2005). Effects of storage time and temperature on egg quality in old laying hens. J. Appl. Poultry Res., 14: 548-553.

Sert D., Aygun A., Demir M.K. (2011). Effects of ultrasonic treatment and storage temperature on egg quality. Poultry Sci., 90: 869-875.

Stepien-Pysniak D. (2010). Occurrence of Gram-negative bacteria in hens' eggs depending on their source and storage conditions. Pol. J. Vet. Sci., 13: 507-513.

Svobodová J., Tůmová E. (2014). Factors affecting microbial contamination of market eggs: a review. Sci. Agric. Bohem., 45: 226-237.

Theron H., Venter P., Lues J.F.R. (2003). Bacterial growth on chicken eggs in various storage environments. Food Res. Int., 36: 969975.

Vlčková J., Tůmová E., Ketta M., Englmaierová M., Chodová D. (2018). Effect of housing system and age of laying hens on eggshell quality, microbial contamination, and penetration of microorganisms into eggs. Czech J. Anim. Sci., 63: 51-60.

Vlčková J., Tůmová E., Míková K., Englmaierová M., Okrouhlá M., Chodová D. (2019). Changes in the quality of eggs during storage depending on the housing system and the age of hens. Poultry Sci., 98: 6187-6193.

Yamak U.S., Boz M.A., Ucar A., Sarcia M., Onder H. (2016). The effect of eggshell thickness on the hatchability of guinea fowl and pheasants. Rev. Bras. Cienc. Avic., 18: 49-53.

You S.J., Udenigwe C.C., Aluko R.E., Wu J. (2010). Multifunctional peptides from egg white lysozyme. Food Res. Int., 43: $848-855$.

Zaheer K. (2015). An updated review on chicken eggs: production, consumption, management aspects and nutritional benefits to human health. Food Nutr. Sci., 6: 1208-1220.

Zita L., Tůmová E., Štolc L. (2009). Effects of genotype, age and their interaction on egg quality in brown-egg laying hens. Acta Vet. Brno, 78: 85-91.

Zita L., Jeníková M., Härtlová H. (2018). Effect of housing system on egg quality and the concentration of cholesterol in egg yolk and blood of hens native resources of the Czech Republic and Slovakia. J. Appl. Poultry Res., 3: 380-388.

Received: 7 I 2021

Accepted: 15 VII 2021 